\title{
Swinging and Tumbling of Fluid Vesicles in Shear Flow
}

\author{
Hiroshi Noguchi and Gerhard Gompper \\ Institut für Festkörperforschung, Forschungszentrum Jülich, 52425 Jülich, Germany
}

(Received 14 November 2006; published 21 March 2007)

\begin{abstract}
The dynamics of fluid vesicles in simple shear flow is studied using mesoscale simulations of dynamically triangulated surfaces, as well as a theoretical approach based on two variables: a shape parameter and the inclination angle, which has no adjustable parameters. We show that, between the wellknown tank-treading and tumbling states, a new "swinging" state can appear. We predict the dynamic phase diagram as a function of the shear rate, the viscosities of the membrane and the internal fluid, and the reduced vesicle volume. Our results agree well with recent experiments.
\end{abstract}

The dynamics of deformable objects such as liquid droplets [1,2], lipid vesicles [3-10], red blood cells [1114], and elastic capsules [15-17] in flows has received increasing attention experimentally, theoretically, and numerically in recent years. All of these objects show phenomenologically similar behaviors in shear flows-either a tank-treading rotation with a stationary shape and a finite inclination angle $\theta>0$ with respect to the flow direction or an unsteady tumbling motion. However, the qualitative and quantitative behavior of the various objects can be very different, because the energies governing their shapes and thermal fluctuations are very different. In the case of fluid vesicles, which we want to investigate in this Letter, the membrane conformations are determined by the curvature elasticity together with the constraints of membrane incompressibility and constant internal volume.

In simple shear flows, with flow velocity $\mathbf{v}=\dot{\gamma} y \mathbf{e}_{x}$, a transition occurs from tank treading to tumbling with an increasing viscosity of the internal fluid $\eta_{\text {in }}[4,8,12]$ or membrane viscosity $\eta_{\mathrm{mb}}[9,10]$. This transition is described very well by the theory of Keller and Skalak (KS) [12], which assumes a fixed ellipsoidal vesicle shape. However, shear forces can be large enough to induce shape transformations of fluid vesicles, for example, from discocyte to prolate at small membrane viscosity and small viscosity contrast between inside and outside or from prolate to discocyte at larger membrane viscosities $[9,10]$. In this case, it is essential to take the membrane deformability into account.

Recently, Kantsler and Steinberg [4] reported the first observation of a new type of vesicle dynamics in shear flow, which is characterized by oscillations of the inclination angle $\theta$ and the deformation, where $-\theta_{0} \lesssim \theta(t) \lesssim \theta_{0}$, with $\theta_{0} / \pi<1$ and time average $\langle\theta\rangle \simeq 0$. The vesicles were found to transit from tumbling to this oscillatory motion with increasing shear rate $\dot{\gamma}$. It is worth mentioning that such an oscillatory motion was also observed in our previous simulations (see Fig. 6 in Ref. [10]) but not further analyzed. Simultaneously with the experiment, Misbah [6] predicted a "vacillating breathing" mode for quasispherical fluid vesicles. This mode exhibits similar dynamical behavior as seen experimentally; however, it "coexists" with the tumbling mode, and its orbit depends on the initial deformation; i.e., it is not a limit cycle. Furthermore, the shear rate appears only as the basic time scale and, therefore, cannot induce any shape transitions. Hence, it does not explain the tumbling-to-oscillatory transition seen in the experiments [4].

In this Letter, we study the oscillatory dynamics of fluid vesicles, which we will refer to as the "swinging mode," by mesoscale hydrodynamics simulations and a simplified nonlinear theoretical model. The main questions we want to address are as follows: How does the bending energy affect the dynamics? Can transitions between modes be induced by varying the shear rate? What happens beyond the quasispherical limit, which is the typical experimental situation? What is the effect of the membrane viscosity? Is the swinging mode stable when thermal membrane undulations are taken into account? We will show that the experiments of Ref. [4] can be understood very well on the basis of our theory. Furthermore, we will present a complete phase diagram of vesicle motion as a function of shear rate and viscosity.

It is worth mentioning that elastic capsules [15] and red blood cells [18] can also exhibit a swinging mode; however, in this case, the angle $\theta(t)$ is always positive during the oscillation. Very recently, this dynamics was explained by the KS theory with an addition of an energy barrier for the tank-treading rotation caused by the membrane shear elasticity [16]. Therefore, this mechanism cannot be employed to explain the swinging mode of fluid vesicles.

The vesicle dynamics is described by several dimensionless quantities. For a vesicle of volume $V$ and surface area $S$, the reduced volume $V^{*}$ and the excess area $\Delta_{S}$ are defined by $V^{*}=\left(R_{V} / R_{S}\right)^{3}=\left(1+\Delta_{S} / 4 \pi\right)^{-3 / 2}$ and $\Delta_{S}=S / R_{V}^{2}-4 \pi$, where $R_{V}=(3 V / 4 \pi)^{1 / 3}$ and $R_{S}=$ $(S / 4 \pi)^{1 / 2}$. The relative viscosity of the inside fluid and membrane are $\eta_{\mathrm{in}}^{*}=\eta_{\mathrm{in}} / \eta_{0}$ and $\eta_{\mathrm{mb}}^{*}=\eta_{\mathrm{mb}} / \eta_{0} R_{S}$, respectively, where $\eta_{0}$ is the viscosity of the outside fluid. The shape relaxation time of vesicles with bending rigidity $\kappa$ is given by $\tau=\eta_{0} R_{S}^{3} / \kappa$ (for $\eta_{\text {in }}^{*}=1$ ). This time is used to define a reduced shear rate $\dot{\gamma}^{*}=\dot{\gamma} \tau$. 
The hydrodynamics of fluid vesicle can be studied very well by hybrid simulations of a dynamically triangulated membrane model [19] and a particle-based mesoscale solvent, multiparticle collision dynamics [20]. A detailed description of this method can be found in Ref. [10].

We have simulated a prolate vesicle with a viscous membrane at $V^{*}=0.78$ and $\eta_{\text {in }}^{*}=1$. Figure 1 shows the time evolution of the vesicle shape and $\theta$. The shape parameter is $\alpha_{D}=\left(L_{1}-L_{2}\right) /\left(L_{1}+L_{2}\right)$, where $L_{1}$ and $L_{2}$ are the maximum lengths in the direction of the eigenvectors of the gyration tensor in the vorticity $(x, y)$ plane. The vesicle is found to exhibit a tumbling motion for $\dot{\gamma}^{*}=$ 0.92 but a swinging motion for $\dot{\gamma}^{*}=3.68$. As $\dot{\gamma}^{*}$ increases, the tumbling frequency $f_{\text {tmb }}$ decreases (see Fig. 2), while the frequency of the swinging motion increases. The shear rate $\dot{\gamma}^{*}$ at the tumbling-to-swinging transition increases with increasing $\eta_{\mathrm{mb}}^{*}$. These results are consistent with the experiments of Ref. [4].

In order to obtain a better understanding of the vesicle dynamics, we now derive an approximate theoretical model. First, we follow Refs. [5,6] and employ the Stokes approximation and perturbation theory for quasispherical vesicles. The vesicle shape is expanded in spherical harmonics $Y_{l, m}$ as $R=R_{V}\left(1+\sum_{l, m} u_{l, m} Y_{l, m}\right)$. The free energy of the membrane with bending rigidity $\kappa$ and surface tension $\sigma$ is $F=\int d S\left\{\sigma+(\kappa / 2)\left(C_{1}+C_{2}\right)^{2}\right\}$, where $C_{1}$ and $C_{2}$ are the principal curvatures at each point of the membrane. With the harmonic approximation, it is given by $F=(\kappa / 2) \sum_{l, m} E_{l}\left|u_{l, m}\right|^{2}+O\left(u_{l, m}^{3}\right)$, where $E_{l}=(l+2) \times$
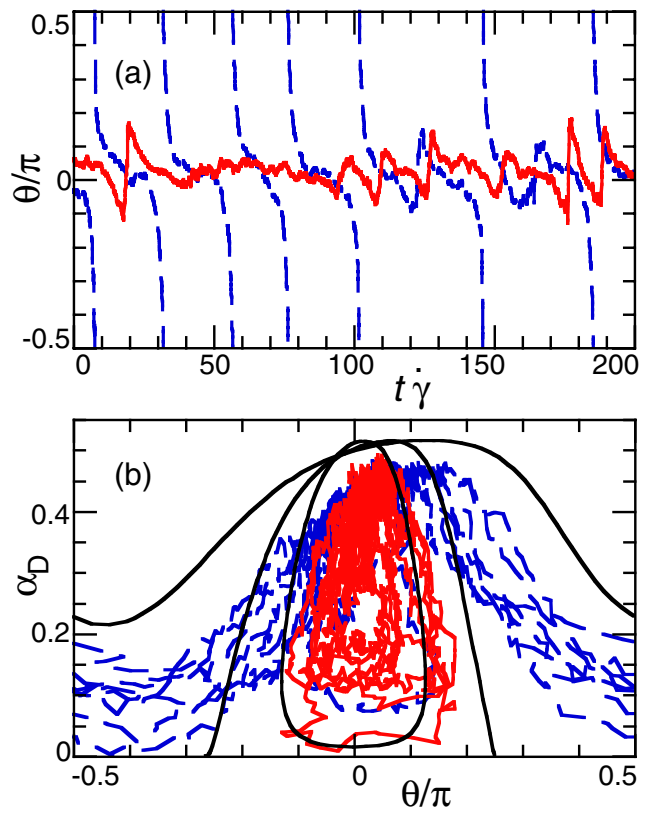

FIG. 1 (color online). Temporal evolution of $\alpha_{D}$ and $\theta$, for $V^{*}=0.78$ and $\eta_{\mathrm{mb}}^{*}=2.9$. The solid (red) and dashed (blue) lines represent simulation data for $\dot{\gamma}^{*}=3.68$ and $0.92\left(\kappa / k_{B} T=\right.$ 10 and 40 , with $\dot{\gamma} \eta_{0} R_{S}^{3} / k_{B} T=36.8$ ), respectively. The solid lines in (b) are obtained from Eqs. (6) and (7) without thermal noise for $\dot{\gamma}^{*}=1.8,3$, and 10 (from top to bottom). $(l-1)\left\{l(l+1)+\sigma R_{V}^{2} / \kappa\right\}$. The flow fields inside and outside of the vesicle are described by the Lamb solution [5]. The flow stress on the membrane is balanced with the elastic forces due to bending and tension. This implies that the undulation amplitudes $u_{l, m}$ are determined by

$$
\frac{\partial u_{l, m}}{\partial t}=\frac{i \dot{\gamma} m}{2} u_{l, m}-\frac{\kappa \Gamma_{l} E_{l}}{2 \eta_{0} R_{V}^{3}} u_{l, m} \mp i h \dot{\gamma} \delta_{l, 2} \delta_{m, \pm 2},
$$

where $h=60 \sqrt{2 \pi / 15} /\left(32+23 \eta_{\text {in }}^{*}\right) \quad$ and $\quad \Gamma_{l}=l(l+$ 1) $/\left\{(l+2)\left(2 l^{2}-l+2\right)+(l-1)\left(2 l^{2}+5 l+5\right) \eta_{\text {in }}^{*}\right\}$. The detailed derivation of Eq. (1) for $\eta_{\text {in }}^{*}=1$ is described in Ref. [5].

In this Letter, we focus on vesicles, which are symmetric with respect to the vorticity plane through their center. Hence, we consider only $u_{2, \pm 2}$ and $u_{2,0}$ and decompose $u_{2, \pm 2}$ into amplitude and phase: $u_{2, \pm 2}=r \exp (\mp 2 i \theta)$, where $\theta$ corresponds to the inclination angle [6]. Since the curvature energy does not contribute in this case, we have to go beyond the harmonic approximation. Therefore, we replace the force $\kappa E_{l} r$ in Eq. (1) by $\partial F / \partial r$. This implies

$$
\begin{gathered}
\frac{\partial r}{\partial t}=-\frac{\Gamma_{2}}{2 \eta_{0} R_{V}^{3}}\left(\frac{\partial F}{\partial r}+2 \lambda r\right)+\dot{\gamma} h \sin (2 \theta), \\
\frac{\partial \theta}{\partial t}=\frac{\dot{\gamma}}{2}\left\{-1+\frac{h}{r} \cos (2 \theta)\right\} \\
\frac{\partial u_{2,0}}{\partial t}=-\frac{\Gamma_{2}}{\eta_{0} R_{V}^{3}}\left(\frac{\partial F}{\partial u_{2,0}}+\lambda u_{2,0}\right) .
\end{gathered}
$$

A Lagrange multiplier $\lambda \equiv \sigma R_{V}^{2}$ is employed to satisfy the area constraint [21]; it is determined by $d \Delta_{S} / d t=0$, which implies $\Delta_{S}=2 u_{2,0}^{2}+4 r^{2}$. Thus, Eq. (2) becomes

$$
\frac{d r}{d t}=\left(1-\frac{4 r^{2}}{\Delta_{S}}\right)\left\{-\left.\frac{\Gamma_{2}}{2 \eta_{0} R_{V}^{3}} \frac{\partial F}{\partial r}\right|_{\Delta_{S}}+\dot{\gamma} h \sin (2 \theta)\right\},
$$

where $\partial F /\left.\partial r\right|_{\Delta_{S}}=\partial F / \partial r-\left(2 r / u_{2,0}\right) \partial F / \partial u_{2,0}$. In the harmonic approximation of $F$, the first term in Eq. (5)

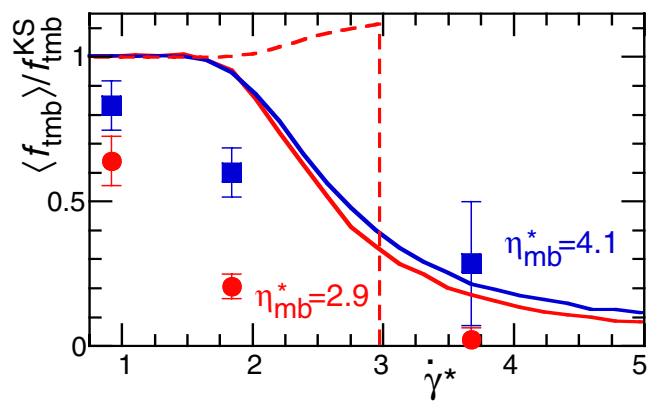

FIG. 2 (color online). $\quad \dot{\gamma}$ dependence of the tumbling frequency $f_{\text {tum }}$ normalized by the frequency $f_{\text {tum }}^{\mathrm{KS}}=\dot{\gamma} \sqrt{1-B^{2}} / 2 \pi$ of KS theory at $V^{*}=0.78$. The bending rigidity $\kappa$ is varied to change $\dot{\gamma}^{*}$, with $\dot{\gamma} \eta_{0} R_{S}^{3} / k_{B} T=36.8$. The symbols represent simulation data for $\eta_{\mathrm{mb}}^{*}=2.9(\mathbf{O})$ and $4.1(\boldsymbol{\square})$. The solid and dashed lines are obtained from Eqs. (6) and (7) with and without thermal noise, respectively. 
disappears because $\partial F /\left.\partial r\right|_{\Delta_{S}}=0$, so that we recover the description of Ref. [6]. The prolate shape appears as an energy minimum, when higher-order terms in the free energy $F$ are taken into account [21].

Since an expansion of the vesicle shape in spherical harmonics is difficult experimentally, more easily accessible measures of the deformation are desirable. Furthermore, a description is needed which goes beyond the quasispherical limit. Thus, instead of $r$, we employ the shape parameter $\alpha_{D}=\left(L_{1}-L_{2}\right) /\left(L_{1}+L_{2}\right)$, which is easily measurable by microscopy. Since $\alpha_{D}=$ $(\sqrt{15 / 2 \pi} / 2) r+O\left(r^{2}\right)$, Eq. (5) implies

$$
\frac{d \alpha_{D}}{d t}=\left\{1-\left(\frac{\alpha_{D}}{\alpha_{D}^{\max }}\right)^{2}\right\}\left\{-\frac{A_{0}}{\tau \kappa V^{*}} \frac{\partial F}{\partial \alpha_{D}}+\dot{\gamma} A_{1} \sin (2 \theta)\right\},
$$

where $A_{0}=15 \Gamma_{2} / 16 \pi=45 / 8 \pi\left(32+23 \eta_{\text {in }}^{*}\right)$ and $A_{1}=$ $h \sqrt{15 / 2} \pi / 2=30 /\left(32+23 \eta_{\text {in }}^{*}\right)$. Since an accurate evaluation of $F$ is very important, we calculate it numerically for ellipsoidal vesicles with $\left(x_{1} / a_{1}\right)^{2}+\left(x_{2} / a_{2}\right)^{2}+\left(x_{3} / a_{3}\right)^{2}=$ 1; see the inset in Fig. 3. The prolate $\left(a_{1}>a_{2}=a_{3}\right)$ and oblate $\left(a_{1}=a_{2}>a_{3}\right)$ shapes are energy minima and maxima, respectively, and $\partial F / \partial \alpha_{D}$ diverges in the limit of maximum extension $\alpha_{D} \rightarrow \alpha_{D}^{\max }\left(V^{*}\right)$. Equation (6) has the same form as the simplified model studied previously $[9,10]$ but now has no adjustable parameters.

In a final step, in order to obtain a reliable description also for large excess areas, we replace Eq. (3) by the equation of KS theory [12,13], which reads

$$
\begin{aligned}
\frac{d \theta}{d t} & =\frac{\dot{\gamma}}{2}\{-1+B \cos (2 \theta)\}, \\
B & =f_{0}\left\{f_{1}+\frac{f_{1}^{-1}}{1+f_{2}\left(\eta_{\mathrm{in}}^{*}-1\right)+f_{2} f_{3} \eta_{\mathrm{mb}}^{*}}\right\},
\end{aligned}
$$

where the factors $f_{0}, f_{1}, f_{2}$, and $f_{3}$ are the functions of the ellipsoidal shape $\left(a_{2} / a_{1}, a_{3} / a_{1}\right)$ and are given in Appendix B of Ref. [10]. The KS theory, in general, shows

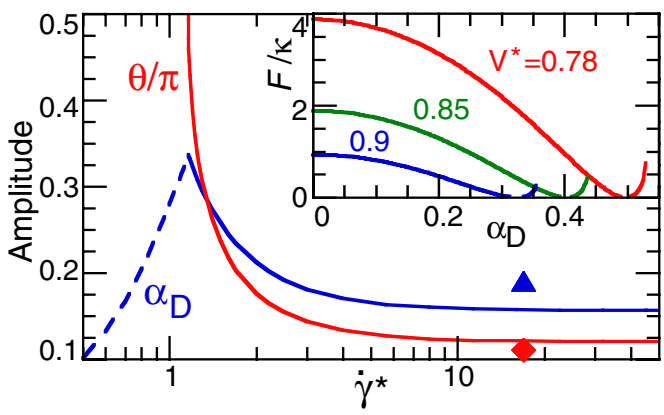

FIG. 3 (color online). $\quad \dot{\gamma}$ dependence of the amplitudes of the oscillations of $\alpha_{D}$ and $\theta$ for $V^{*}=0.9, \eta_{\mathrm{in}}^{*}=6$, and $\eta_{\mathrm{mb}}^{*}=0$. The dashed and solid lines represent the tumbling and swinging states, respectively, given by Eqs. (6) and (7) without thermal noise. Symbols represent estimates from the experimental data [4] for $\alpha_{D}(\mathbf{\Delta})$ and $\theta(\bullet)$. The inset shows the curvature energy $F\left(\alpha_{D}\right)$ of ellipsoids, relative to the prolate shape, for $V^{*}=0.78$, 0.85 , and 0.9 . very good agreement with simulation results $[7,10]$. When thermal fluctuations are taken into account, Gaussian white noises $g_{\alpha}(t)$ and $g_{\theta}(t)$ are added to Eqs. (6) and (7), respectively, which obey the fluctuation-dissipation theorem, so that $\left\langle g_{i}(t)\right\rangle=0,\left\langle g_{i}(t) g_{j}\left(t^{\prime}\right)\right\rangle=2 k_{B} T / \zeta_{i} \delta_{i j} \delta(t-$ $\left.t^{\prime}\right)$, where $i, j \in\left\{\alpha_{D}, \theta\right\}$. The friction coefficients are $\zeta_{\alpha}=$ $\tau \kappa V^{*} / A_{0}\left\{1-\left(\alpha_{D} / \alpha_{D}^{\max }\right)^{2}\right\}$ and the rotational friction coefficient of a sphere $\zeta_{\theta}=8 \pi \eta_{0} R_{S}^{3}$. We numerically integrate Eqs. (6) and (7) with or without thermal noise using the second- or fourth-order Runge-Kutta method, respectively. When $\alpha_{D}(t)$ becomes zero, we set $\left(\alpha_{D}, \theta\right)=$ $(0, \pi / 4)$, since $\theta=\pi / 4$ is the inclination angle in the spherical limit.

Equations (6) and (7) reproduce the simulation results semiquantitatively (see Figs. 1 and 2). This is a very good agreement, given the fact that our model certainly does not systematically take into account all higher-order terms and, furthermore, has no adjustable parameters. At small $\dot{\gamma}^{*}$, in the tumbling phase, $\theta$ rotates with the $\alpha_{D}$ oscillation of small amplitude. This $\alpha_{D}$ amplitude becomes larger at larger $\dot{\gamma}^{*}$; see Fig. 3. Then, when $\alpha_{D}(t)$ reaches zero, $\theta$ jumps to $\pi / 4$; see Fig. 1 . This type of $\theta$ oscillation with a jump is reminiscent of the behavior predicted for very viscous liquid drops within perturbation theory [2]. Finally, at even larger $\dot{\gamma}^{*}, \alpha_{D}$ and $\theta$ exhibit oscillations without jumps.

The physical mechanism of swinging can be understood on the basis of Eqs. (6) and (7) as follows. At finite $\kappa$, the shear force elongates the vesicle $\left(\alpha_{D}\right.$ increases) for $0<$ $\theta<\pi / 2$ but compresses it ( $\alpha_{D}$ decreases) for $-\pi / 2<$ $\theta<0$, since the $\sin (2 \theta)$ term in Eq. (6) changes sign. Thus, the swinging motion is caused by a shape deformation, where $B$ in Eq. (7) crosses the tank-treading-to-tumbling threshold periodically. First, a prolate vesicle starts tumbling because $B<1$, and $\alpha_{D}$ decreases when $\theta<0$, which implies that $B$ increases; then $\theta$ starts to increase again because $B>1$ at small $\alpha_{D}$; finally, $\alpha_{D}$ increases when $\theta>$ 0 . In the swinging phase, the amplitudes of $\alpha_{D}$ and $\theta$ decrease and saturate to finite values with increasing $\dot{\gamma}^{*}$; see Fig. 3.

A linear stability analysis of the fixed points of Eqs. (6) and (7) shows that the tank-treading-to-tumbling transition always occurs as a saddle-node bifurcation at $\theta \simeq 0$. The tank-treading-to-swinging transition is a saddle-node bifurcation for small $\dot{\gamma}^{*}$ but becomes a Hopf bifurcation at $\theta<0$ for larger $\dot{\gamma}^{*}$. Near the boundary of these two bifurcations, a second range of stable fixed points appears between the saddle and unstable fixed points at $\theta<0$. However, these stable points at $\theta<0$ will likely disappear when the full angular degrees of freedom are taken into account, since they should be unstable in the vorticity direction.

Figure 4 shows the full phase diagrams, for both variations of the internal and the membrane viscosities. With increasing $V^{*}$, the smallest shear rate $\dot{\gamma}_{\mathrm{os}}^{*}$, at which swinging can be observed, decreases, since the dependence of the 

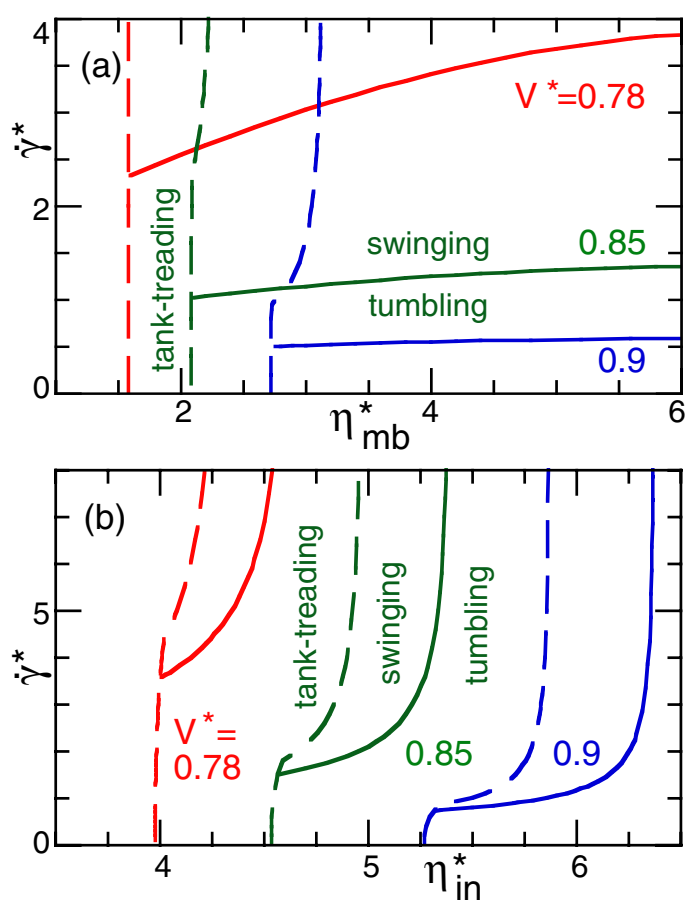

FIG. 4 (color online). Dynamical phase diagrams as a function of (a) $\eta_{\mathrm{mb}}^{*}$ for $\eta_{\mathrm{in}}^{*}=1$, and (b) $\eta_{\mathrm{in}}^{*}$ for $\eta_{\mathrm{mb}}^{*}=0$, for various reduced volumes $V^{*}$, obtained from Eqs. (6) and (7) without thermal noise. The tank-treading phase is located on the lefthand side of the dashed lines. The solid lines represent the tumbling-to-swinging transitions.

energy $F$ on $\alpha_{D}$ becomes more shallow; compare Fig. 3. Also, the swinging region shifts to higher viscosity, $\eta_{\text {in }}$ or $\eta_{\mathrm{mb}}$, with increasing $V^{*}$. On the other hand, for fixed $V^{*}$, $\dot{\gamma}_{\mathrm{os}}^{*}$ is a strongly increasing function of $\eta_{\mathrm{in}}^{*}$, while the increase with $\eta_{\mathrm{mb}}^{*}$ is much less pronounced. At $\eta_{\mathrm{mb}} \rightarrow$ $\infty$, however, any vesicle becomes solidlike and should exhibit a tumbling motion. The reason for this discrepancy is that, in the derivation of Eq. (1), we have neglected the dependence on $\eta_{\mathrm{mb}}^{*}$. In analogy with Eq. (7), we expect that in a more detailed calculation a linear combination of $\eta_{\mathrm{mb}}^{*}$ and $\eta_{\text {in }}^{*}$ will appear in both $h$ and $\Gamma_{l}$-and thus also in $A_{0}$ and $A_{1}$ in Eq. (6). These additional $\eta_{\mathrm{mb}}^{*}$ contributions should increase the slope of the $\dot{\gamma}_{\text {os }}^{*}$ lines in Fig. 4(a); this is also consistent with the strong $\eta_{\mathrm{mb}}^{*}$ dependence seen in the simulations; see Fig. 2.

In the experiments of Ref. [4], the swinging (tumbling) motion was observed for $\dot{\gamma}^{*} \simeq 17$ (4.5) [22], $\eta_{\text {in }}^{*}=6$ (8.4), $V^{*} \simeq 0.9$, and a very small membrane viscosity $\eta_{\mathrm{mb}}^{*} \sim 0.1$ (calculated from $\eta_{\mathrm{mb}} \sim 10^{-9} \mathrm{Ns} / \mathrm{m}$ of Ref. [23]). The swinging motion is observed in close vicinity to the tanktreading-to-tumbling transition [4]. Thus, the experimental data agree with our predicted phase diagram (Fig. 4) very well. Furthermore, the oscillation amplitudes also show good agreement; see Fig. 3 for swinging. For tumbling at $\dot{\gamma}^{*} \simeq 4.5$ and $\eta_{\text {in }}^{*}=8.4$, the amplitudes $\alpha_{D}^{\mathrm{amp}}=0.122$ and 0.13 are found in theory and experiment, respectively.

In summary, we have studied the oscillatory motion of fluid vesicles in simple shear flow. We have developed a simplified model for the ellipsoidal fluid vesicle, which explains the simulation and experimental results very well. This model can be applied to other shapes, such as discocytes at $V^{*} \simeq 0.6$, where $F\left(\alpha_{D}\right)$ has to be calculated from simulations [10]. In the future, it will be interesting to investigate the coupling of different oscillation mechanisms in composite membranes such as red blood cells.

We thank R. Finken (Stuttgart) for his help with rederiving Eq. (1). G.G. acknowledges support of this work through the DFG priority program "Nano- and Microfluidics."

*Electronic address: hi.noguchi@fz-juelich.de

[1] J. M. Rallison, Annu. Rev. Fluid Mech. 16, 45 (1984).

[2] D. Barthès-Biesel and H. Sgaier, J. Fluid Mech. 160, 119 (1985).

[3] K. H. de Haas, C. Blom, D. van den Ende, M. H. G. Duits, and J. Mellema, Phys. Rev. E 56, 7132 (1997).

[4] V. Kantsler and V. Steinberg, Phys. Rev. Lett. 96, 036001 (2006); 95, 258101 (2005).

[5] U. Seifert, Eur. Phys. J. B 8, 405 (1999).

[6] C. Misbah, Phys. Rev. Lett. 96, 028104 (2006).

[7] M. Kraus, W. Wintz, U. Seifert, and R. Lipowsky, Phys. Rev. Lett. 77, 3685 (1996).

[8] J. Beaucourt, F. Rioual, T. Séon, T. Biben, and C. Misbah, Phys. Rev. E 69, 011906 (2004).

[9] H. Noguchi and G. Gompper, Phys. Rev. Lett. 93, 258102 (2004).

[10] H. Noguchi and G. Gompper, Phys. Rev. E 72, 011901 (2005).

[11] T. M. Fischer, M. Stöhr-Liesen, and H. SchmidSchönbein, Science 202, 894 (1978).

[12] S. R. Keller and R. Skalak, J. Fluid Mech. 120, 27 (1982).

[13] R. Tran-Son-Tay, S. P. Sutera, and P. R. Rao, Biophys. J. 46, 65 (1984).

[14] C. Pozrikidis, Ann. Biomed. Eng. 31, 1194 (2003).

[15] K. S. Chang and W. L. Olbricht, J. Fluid Mech. 250, 609 (1993); S. Ramanujan and C. Pozrikidis, J. Fluid Mech. 361, 117 (1998).

[16] J. M. Skotheim and T. W. Secomb, Phys. Rev. Lett. 98, 078301 (2007).

[17] R. Finken and U. Seifert, J. Phys. Condens. Matter 18, L185 (2006).

[18] M. Abkarian, M. Faivre, and A. Viallat (unpublished).

[19] G. Gompper and D. M. Kroll, J. Phys. Condens. Matter 9, 8795 (1997).

[20] A. Malevanets and R. Kapral, J. Chem. Phys. 110, 8605 (1999).

[21] S. T. Milner and S. A. Safran, Phys. Rev. A 36, 4371 (1987).

[22] The reduced shear rate is defined differently in Ref. [4].

[23] R. Dimova, C. Dietrich, A. Hadjiisky, K. Danov, and B. Pouligny, Eur. Phys. J. B 12, 589 (1999). 